\title{
Nab-Paclitaxel for Metastatic Pancreatic Cancer: Clinical Outcomes and Potential Mechanisms of Action
}

\author{
Salah-Eddin Al-Batran ${ }^{\mathrm{a}}$ Michael Geissler ${ }^{\mathrm{b}}$ Thomas Seufferlein $^{\mathrm{c}}$ Helmut Oettle $^{\mathrm{d}}$ \\ ${ }^{a}$ Krankenhaus Nordwest, UCT University Cancer Center Frankfurt, \\ ${ }^{\mathrm{b}}$ Klinik für Allgemeine Innere Medizin, Onkologie/Hämatologie, Gastroenterologie und Infektiologie, Klinikum Esslingen, \\ ${ }^{c}$ Klinik für Innere Medizin I, Universitätsklinikum UIm, \\ ${ }^{\mathrm{d} O n k o l o g i s c h e ~ S c h w e r p u n k t p r a x i s, ~ F r i e d r i c h s h a f e n, ~ G e r m a n y ~}$
}

\section{Keywords}

Pancreatic cancer · Chemotherapy · Nab-paclitaxel Stroma SPARC

\section{Summary}

For almost 15 years there has been stagnation in the systemic treatment of patients with pancreatic ductal adenocarcinoma (PDAC). Recently, several developments seem to indicate clinically relevant improvements in the treatment of patients with metastatic disease. One of these developments is the introduction of nanoparticle albumin-bound paclitaxel (nab-paclitaxel) into the firstline treatment of metastatic disease. In this review, underlying preclinical and clinical data are discussed, with a special focus on mechanisms of action, the potential interaction with albumin and calcium-binding matricellular glycoproteins, such as the secreted protein acidic and rich in cysteine (SPARC), as well as the clinical outcome associated with the use of nab-paclitaxel.

\section{Introduction}

The management of pancreatic ductal adenocarcinoma (PDAC) remains one of the major challenges in clinical oncology. Pancreatic cancer accounts for roughly $3 \%$ of all newly diagnosed malignancies in developed countries. In 2008, its incidence in Germany was 14,960 cases, and 14,835 patients died from the disease, making pancreatic cancer the fourth most common cause of cancer-related deaths in Germany $[1,2]$. Like many other malignancies, PDAC affects mostly elderly persons. The median age at first diagnosis is 70 for male and 76 years for female patients. Causal factors are environmental and genetic. The most evident environmental factor is tobacco use. Smokers are at a 3.6 times higher risk of developing PDAC than non-smokers [3]. Up to $10 \%$ of PDAC cases show some type of familial aggregation, although the underlying genetic alterations are still not fully understood [4-6]. The majority of patients are diagnosed with an advanced stage of the disease and, overall, more than $95 \%$ of patients diagnosed with PDAC die from their disease within a few months [7]. The high mortality rate is a result of multiple factors including late diagnosis, early systemic spread and a poor response to chemo- and radiotherapy [7].

\section{Systemic Therapy of Metastatic PDAC}

The phase III trial of gemcitabine versus 5-fluorouracil (5-FU) published by Burris and colleagues in 1997 [8] was a landmark trial for the systemic treatment of metastatic PDAC. It changed clinical practice and made the intravenous administration of gemcitabine the standard of care in the first-line setting. Gemcitabine significantly improved the clinical benefit response (primary endpoint of the trial), a composite of pain score, performance status, and weight maintenance. It also resulted in a significant survival benefit of 1.24 months compared to 5-FU (median overall survival (OS) 5.65 vs. 4.41 months, $\mathrm{p}=0.0025)$. 1 -year survival rates were $18 \%$ with gemcitabine and $2 \%$ for 5 -FU. Numerous attempts to build on this standard by combining gemcitabine with 5-FU, irinotecan, platinums, capecitabine, premetrexed, bevacizumab or various biologicals failed in achieving relevant improvements for patients with advanced PDAC, particularly in terms of OS [9-18]. The addition of erlotinib to gemcitabine resulted in a statistically significant, but only marginal improvement in OS and, subsequently, in approval of the combination therapy for first-line treatment. Median OS was 6.24 months with gemcitabine and erlotinib compared with 5.91 months with gemcitabine alone [19]. Recently, substantial progress has been achieved for patients with metastatic PDAC using the triple cytotoxic combination of oxaliplatin, 5-FU, leucovorin

\section{KARGER}

Fax +497614520714

Information@Karger.com

www.karger.com
(C) 2014 S. Karger GmbH, Freiburg

2296-5270/14/0373-0128\$39.50/0

Accessible online at:

www.karger.com/ort
PD Dr. med. Salah-Eddin Al-Batran

Krankenhaus Nordwest

UCT University Cancer Center

Steinbacher Hohl 2-26, 60488 Frankfurt/Main, Germany

info@al-batran.de 
and irinotecan (FOLFIRINOX). FOLFIRINOX significantly improved OS to 11.1 months as compared to 6.8 months for gemcitabine, as well as all other clinically relevant endpoints. However, the regimen represents an intensive combination that is associated with significant toxicities and thus can be only recommended for selected patients of good performance status and no significant co-morbidity [20].

\section{Stroma of Pancreatic Cancers}

PDAC show extensive desmoplasia, a remarkable increase of intratumoral connective tissue surrounding the malignant cells $[21,22]$. Desmoplasia leads to increased interstitial fluid pressure (IFP), which impairs transvascular transport and reduces the delivery of cytotoxic drugs to malignant cells. Moreover, tumor desmoplasia seems to provide an active environment that facilitates tumor progression and metastasis. Recently, targeting the stroma by stromal depletion and disturbing tumor-stroma interaction have been identified as promising therapeutic targets in the treatment of pancreatic cancer [21, 23, 24].

The tumor stroma comprises extracellular matrix (ECM), blood vessels, stromal fibroblasts and immuno-inflammatory cells. Key components for stroma formation are the pancreatic stellate cells. Activated pancreatic stellate cells adopt a myofibroblast-like phenotype and secrete excess amounts of ECM as well as matrix-degrading enzymes and growth factors that facilitate cancer initiation, growth and progression. As a result of perpetuating their own activity via autocrine loops, there is excessive growth of these cells and continuous deposition of ECM [21, 22]. Thus, the stroma of PDAC represents as a dense, fibrotic mass surrounding the tumor cells. Acting as a mechanical barrier to the tumor, it may present a blockade against drug penetration and probably contributes to the observed resistance of PDAC to systemic therapy.

Among the multitude of stromal components the matricellular glycoprotein SPARC (secreted protein acidic and rich in cysteine), an albumin- and calcium-binding glycoprotein, also known as osteonectin or BM-40, has attracted particular interest. It modulates the interaction of cells with the ECM [2527]. SPARC is multifunctional. It plays a key role in tumor growth and metastasis, is involved in enhancement of cell migration and invasive capacity, and is probably implicated in tumor escape by inhibiting immune surveillance and promoting angiogenesis [27-29]. Overexpression of SPARC has been demonstrated in numerous tumor entities and seems to be a prognostic marker of poor survival in certain tumor types [28, 30-33]. In certain tumors, SPARC also has antitumor properties [34]. In pancreatic cancer SPARC is procarcinogenic, making it a target for therapy. SPARC is highly expressed on pancreatic stellate cells and at a reduced level in epithelial tumor cells [27, 34]. Roughly $80 \%$ of PDAC exhibit an increased expression of SPARC in their stroma [33].

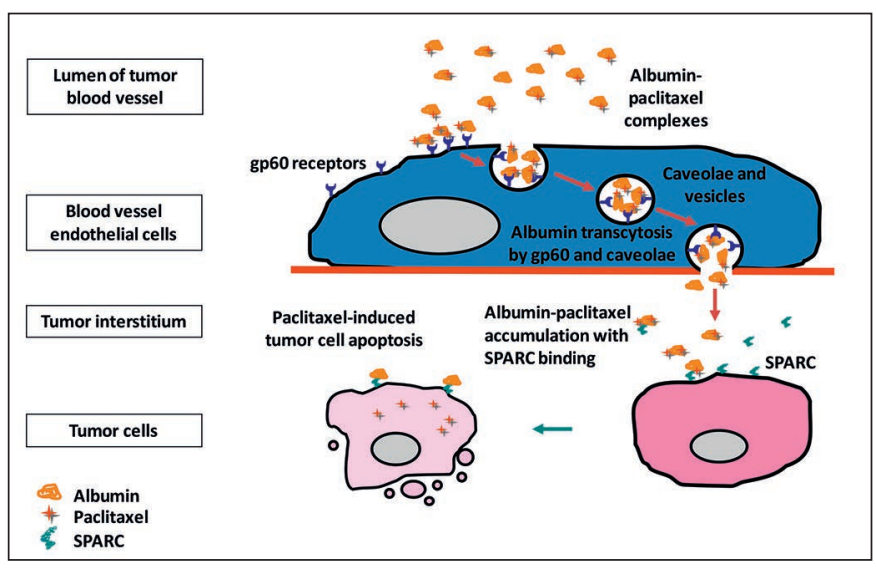

Fig. 1. Proposed mechanism of action of nab-paclitaxel.

From the results of preclinical studies, it has been hypothesized that, in PDAC, SPARC may facilitate the transport of albumin from the ECM to tumor cells. Accumulation of albumin has been found in malignant cells, together with co-localization of SPARC and albumin [35]. Other data suggest that SPARC may mediate the internalization of various molecules in fibroblasts by shuttling between the ECM and the cells [36].

\section{Nab-Paclitaxel}

Nab-paclitaxel is a solvent-free colloidal suspension of paclitaxel and human serum albumin. The nab-platform binds hydrophobic drugs to albumin, thus eliminating the need for toxic solvents. The albumin-bound paclitaxel nanoparticles have an average size of $130 \mathrm{~nm}$ and dissociate in the blood vessel into individual albumin-paclitaxel-complexes of around $10 \mathrm{~nm}$ [37].

Nab-paclitaxel probably exploits the natural features of albumin through 2 mechanisms: utilizing the natural carrier for hydrophobic molecules and its active gp60-receptor-mediated transcytosis across the blood vessel endothelium, and taking advantage of albumin's binding to SPARC for periand intratumoral accumulation of the cytotoxic agent, i.e. albumin-bound paclitaxel [37, 38] (fig. 1). Indeed, preclinical studies demonstrated a roughly 10 -fold higher endothelial binding of nab-paclitaxel and a 4-fold higher rate of transcytosis across endothelial cells compared to solvent-based paclitaxel [39].

Nab-paclitaxel does not require corticosteroid premedication, and can be given as a short infusion over $30 \mathrm{~min}$. Its pharmacokinetics differ considerably from those of solvent-based taxanes. Infusion of nab-paclitaxel results in a higher $\mathrm{C}_{\max }$ and a higher area under the curve (AUC) of free paclitaxel [40]. Nab-paclitaxel exhibits linear pharmacokinetics, allowing for a dose-dependent antitumor activity [41].

Clinical studies showed that nab-paclitaxel was superior to solvent-based paclitaxel in terms of response rates and pro- 
gression-free survival (PFS) in metastatic breast cancer [42] and of response rate in lung cancer [43]. The safety profile was distinct from solvent-based paclitaxel, with nab-paclitaxel exhibiting significantly less neutropenia but moderately more peripheral neuropathy [42]. First results of the international CA033 trial in chemotherapy-naïve patients with stage IV melanoma demonstrated a significant improvement in PFS for nab-paclitaxel versus dacarbazine (4.8 vs. 2.5 months; $\mathrm{p}=0.044)$. OS data are not yet mature [44].

\section{Nab-Paclitaxel - Data from Clinical Trials in Advanced PDAC}

There are a number of early clinical trials with nab-paclitaxel in PDAC. In 19 patients with advanced disease, secondline therapy with nab-paclitaxel was active and well tolerated [45]. 4 phase I trials of nab-paclitaxel/gemcitabine combinations have been reported. 1 study evaluated the maximum tolerated dose (MTD) of the combination in Chinese patients; 1 explored the hepatic arterial infusion of nab-paclitaxel in combination with intravenous gemcitabine and bevacizumab; and 1 investigated the well-tolerated but modestly active triple combination of nab-paclitaxel, gemcitabine and capecitabine [46-48]. Nab-paclitaxel in combination with gemcitabine for metastatic PDAC was first evaluated in a dosefinding phase I/II trial [32]. In this trial, 67 chemo-naive patients with metastatic PDAC were treated with the combination of nab-paclitaxel and gemcitabine. The MTD used $125 \mathrm{mg} / \mathrm{m}^{2}$ nab-paclitaxel and $1,000 \mathrm{mg} / \mathrm{m}^{2}$ gemcitabine, both delivered on days 1,8 , and 15 of a 28 -day cycle. The overall response rate $(\mathrm{ORR})$ at the MTD $(\mathrm{n}=44)$ was $48 \%$. Median OS was 12.2 months and 1-year survival rate $48 \%$. Patients with a complete metabolic response as assessed by positron emission tomography (PET) had longer median OS than those without a complete metabolic response. Similar distributions of ORR and survival were observed when efficacy was analyzed according to the decrease in CA 19-9 levels. SPARC status was evaluated in 36 patients. Median OS of patients with a high expression of SPARC was significantly longer than OS of those in the low-SPARC group (median OS 17.8 vs. 8.1 months, $p=0.043$ ) (fig. 2) [32].

These data provided the rationale for the phase III MPACT trial of nab-paclitaxel in patients with metastatic PDAC. 861 patients with no prior chemotherapy for metastatic disease were randomized. Patients were either treated with the combination of nab-paclitaxel at $125 \mathrm{mg} / \mathrm{m}^{2}$ and gemcitabine at $1,000 \mathrm{mg} / \mathrm{m}^{2}$, both administered on days 1,8 , and 15 of a 28 day cycle or gemcitabine monotherapy at $1,000 \mathrm{mg} / \mathrm{m}^{2}$ weekly for 7 out of 8 weeks in the first cycle and in subsequent cycles on days 1,8 , and 15 of a 28 -day cycle. The primary efficacy endpoint was OS. Secondary objectives included PFS, response rate, disease control rate, and time to treatment failure. Baseline and demographic characteristics were well balanced between the 2 treatment groups. Patients had a median age of 63 years. $60 \%$ of patients had a Karnofsky performance status of $90-100$, and $39 \%$ had a Karnofsky performance status of 70-80. All patients had to have bilirubin levels at or below the upper limit of the normal range.

An overview of the efficacy data of the MPACT trial is provided in table 1 . Treatment with nab-paclitaxel and gemcitabine resulted in a significant survival benefit (fig. 3).

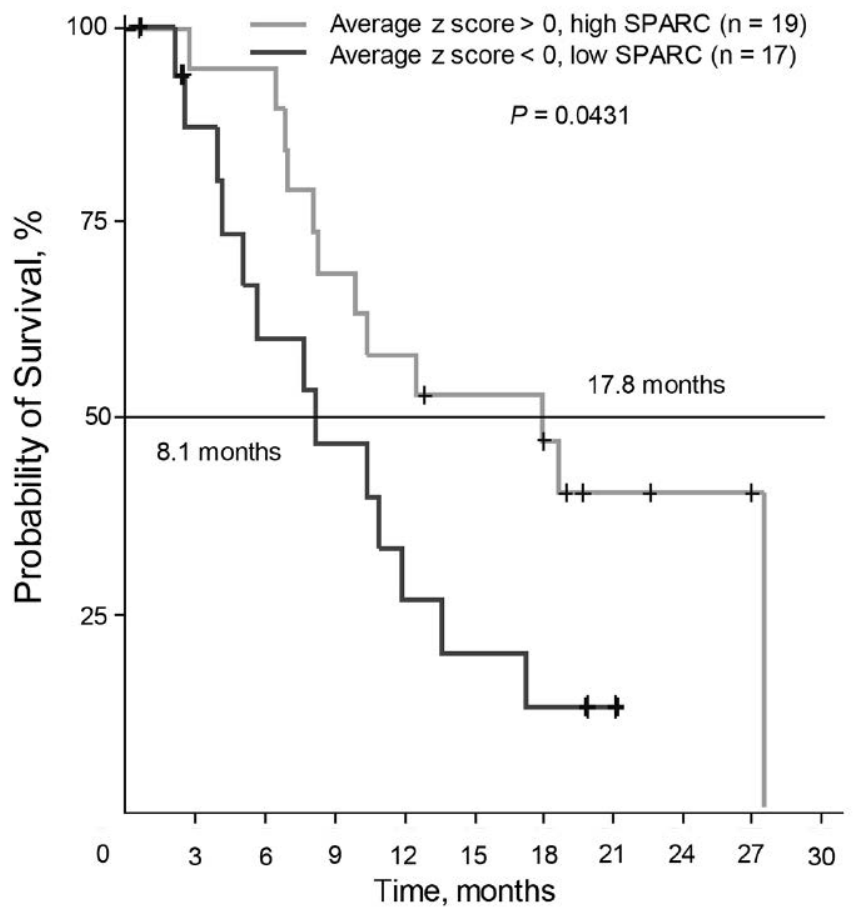

Fig. 2. Correlation between SPARC status and overall survival (OS) in phase I/II trial. OS of 36 patients from all cohorts of the phase I/II trial reported by von Hoff et al. [32], for whom SPARC status was evaluated. Standardized SPARC status was determined via z-score transformation based on measures of maximum intensity, percentage of cells at the maximum intensity and overall score of immunohistochemical staining for SPARC from 7 tissue compartments of a patient's tumor.

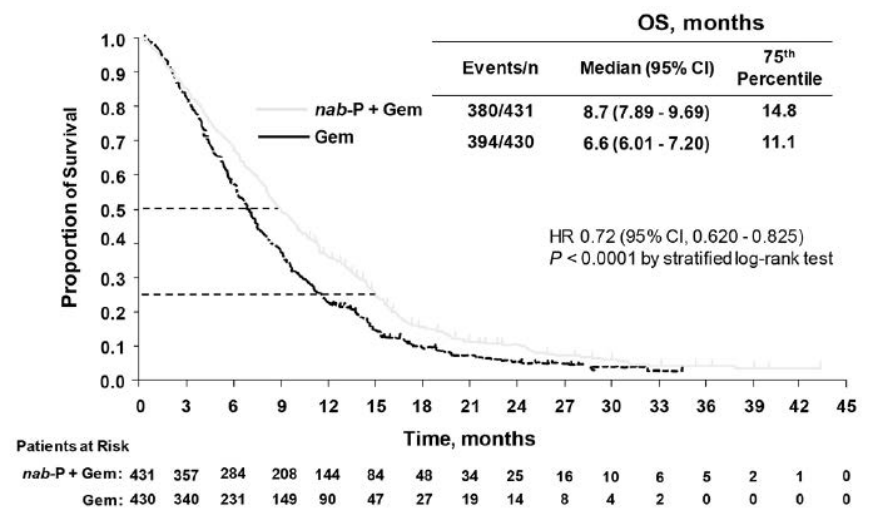

Fig. 3. OS of patients with metastatic pancreatic cancer and first-line therapy in MPACT (intention-to-treat population). Adapted from Goldstein et al. [55]nab-P = nab-paclitaxel, Gem = gemcitabine. 
Table 1. Summary of MPACT efficacy data

\begin{tabular}{|c|c|c|c|c|}
\hline Variable & Nab-paclitaxel and gemcitabine & Gemcitabine & HR or RR & $\mathrm{p}$ value \\
\hline Patients studied, $\mathrm{n}$ & 431 & 430 & & \\
\hline Median OS, months $(95 \% \text { CI })^{\mathrm{a}}$ & $8.7(7.9-9.7)$ & $6.6(6.0-7.2)$ & HR $0.72(0.62-0.83)$ & $<0.001$ \\
\hline \multicolumn{5}{|l|}{ Survival rate, $\%^{\mathrm{b}}$} \\
\hline 6 months & 66 & 55 & n.a. & $<0.001$ \\
\hline 12 months & 35 & 22 & n.a. & $<0.001$ \\
\hline 18 months & 16 & 9 & n.a. & $<0.001$ \\
\hline 24 months & 10 & 5 & n.a. & $<0.001$ \\
\hline PFS, median, months $(95 \% \mathrm{CI})^{\mathrm{b}}$ & $5.5(4.5-5.9)$ & $3.7(3.6-4.0)$ & HR $0.69(0.58-0.82)$ & $<0.001$ \\
\hline ORR by independent review, $\%(95 \% \mathrm{CI})^{\mathrm{b}}$ & $23(19-27)$ & $7(5-10)$ & RR 3.19 (2.18-4.66) & $<0.001$ \\
\hline ORR by investigator review, $\%(95 \% \mathrm{CI})^{b}$ & $29(25-34)$ & $8(5-11)$ & RR $3.81(2.66-5.46)$ & $<0.001$ \\
\hline Disease control rate, $\%(95 \% \mathrm{CI})^{\mathrm{b}}$ & $48(43-53)$ & $33(28-37)$ & RR $1.46(1.23-1.72)$ & $<0.001$ \\
\hline Patients studied ${ }^{\mathrm{b}}, \mathrm{n}$ & 379 & 371 & & \\
\hline$\geq 20 \%$ decrease of CA $19-9, \%$ & 61 & 44 & n.r. & $<0.001$ \\
\hline$\geq 90 \%$ decrease of CA $19-9, \%$ & 31 & 14 & n.r. & $<0.001$ \\
\hline Patients studied ${ }^{c}, \mathrm{n}$ & 130 & 127 & & \\
\hline Metabolic response by PET, \% & 63 & 38 & n.r. & 0.000051 \\
\hline OS, median, months & 10.5 & 8.3 & HR 0.71 & 0.0096 \\
\hline \multicolumn{5}{|c|}{$\begin{array}{l}\mathrm{HR}=\text { hazard ratio, } \mathrm{RR}=\text { response rate ratio, } \mathrm{OS}=\text { overall survival, } \mathrm{PFS}=\text { progression-free survival, } \mathrm{ORR}=\text { overall response rate, } \\
\mathrm{CI}=\text { confidence interval, } \mathrm{PET}=\text { positron emission tomography, } \mathrm{n} . \mathrm{a} .=\text { not applicable, } \text {.r. }=\text { not reported. } \\
\text { a }[55] . \\
\mathrm{b} \text { [ }[49] . \\
\text { c [50]. }\end{array}$} \\
\hline
\end{tabular}

Median OS was increased to 8.7 months (95\% confidence interval (CI) 7.89-9.69) as compared to 6.6 months (95\% CI 6.01-7.20) with gemcitabine alone (hazard ratio (HR) 0.72; $95 \%$ CI $0.62-0.83 ; \mathrm{p}<0.0001)$. 1-year and 2-year survival rates were also significantly higher for the combination $35 \%$ and $10 \%$ for nab-paclitaxel and gemcitabine vs. $22 \%$ and $5 \%$ for gemcitabine alone) [55]. In the multivariate analysis, treatment with the nab-paclitaxel/gemcitabine combination, a favorable performance status, and the absence of liver metastases were independent predictors of favorable survival. Significant benefits for treatment with the combination nabpaclitaxel and gemcitabine over gemcitabine monotherapy were also observed for all secondary endpoints. The effects on OS and PFS were maintained across almost all pre-specified subgroups [49]. Metabolic response evaluated by PET in 257 patients was significantly higher in the combination arm (63\% vs. 38\%, p < 0.001) [50]. CA 19-9 levels decreased (by at least $20 \%$ ) from baseline in $61 \%$ of nab-paclitaxel and gemcitabine-treated patients vs. $44 \%$ of gemcitabine-treated patients $(\mathrm{p}<0.001)$. A decrease of at least $90 \%$ was observed in $31 \%$ vs. $14 \%$ of the treatment groups $(\mathrm{p}<0.001)$, respectively. The decrease in CA 19-9 levels seems to be prognostic of patient's outcome. Patients with $\geq 90 \%$ CA 19-9 decrease had a median OS of 13.5 vs. 8.2 months for those with a decrease of $<90 \%$ (HR 0.53: 95\% CI 0.43-0.67; p < 0.001) [49].

The combination arm was associated with grade 3 or higher neutropenia (38\% for nab-paclitaxel and gemcitabine vs. $27 \%$ for gemcitabine alone) and leukopenia (31\% vs. 16\%), whereas no major differences were observed for febrile neutropenia ( $3 \%$ vs. $1 \%)$, thrombocytopenia (13\% vs. $9 \%$ ), or anemia (13\% vs. $12 \%)$. Receipt of growth factors was $26 \%$ in the combination arm and $15 \%$ in the gemcitabine alone arm. In terms of non-hematological adverse events, the addition of nab-paclitaxel increased the rates of grade $3 / 4$ fatigue $(17 \%$ vs. $7 \%$ ), and grade 3 neuropathy (17\% vs. $1 \%)$. Median time to onset of grade 3 neuropathy with nab-paclitaxel was around 4.5 months. In the combination arm, neuropathy of grade 3 improved to grade 1 or lower in a median of 29 days. Of the patients who had grade 3 peripheral neuropathy, $44 \%$ resumed nab-paclitaxel at a reduced dose [49].

\section{Potential Underlying Mechanisms for the Activity of Nab-Paclitaxel in Pancreatic Cancer}

Nab-paclitaxel's linear pharmacokinetics, its distribution properties into the tumor microenvironment utilizing albumin as a carrier protein, and synergistic effects with gemcitabine represent mechanisms that may explain an activity in PDAC that has not been observed yet with other taxanes.

\section{Potential Interaction of SPARC and Nab-Paclitaxel}

It has been speculated that SPARC expression is implicated in the antitumor activity of albumin-bound drugs. However, the role of SPARC as a target of nab-paclitaxel is still not fully understood [34]. Pharmacokinetic and pharmacodynamic studies in genetically engineered mouse models, including a SPARC-deficient mouse model of PDAC, revealed significantly decreased paclitaxel concentrations in SPARC null tissues when nab-paclitaxel was administered at a low dose [6], pointing towards an interaction between nab-paclitaxel and SPARC. However, this effect disappeared when higher doses (MTD) of mouse nab-paclitaxel were administered, demonstrating that SPARC can be saturated.

In pancreatic cancer patients treated with nab-paclitaxel, Von Hoff et al. [32] observed a significantly longer median OS 
in patients whose tumors had high expression of SPARC as compared to patients with low SPARC expression. SPARC expression significantly predicted a patient's outcome in the multivariate analysis. The expression of SPARC was determined by a composite score from tumor and stromal compartment. While stromal SPARC was significantly correlated with OS, tumoral SPARC was not. Other reports also suggested that the role of SPARC as a prognostic marker for OS in pancreatic cancer may be limited to its expression in the tumor stroma [33]. Since all patients had received nab-paclitaxel in the study mentioned above, it was not possible to distinguish prognostic from predictive effects of SPARC. However, prospective data, as expected from the MPACT trial, are needed for clarification of the correlation of SPARC and outcomes following therapy with nab-paclitaxel.

\section{Depletion of Tumor Stroma}

It has been shown that the treatment with nab-paclitaxel affects the stroma of PDAC. In a mouse model, tumor stromal density, measured through reduction in -smooth muscle actin and collagen 1 expression, was decreased by nab-paclitaxel [52]. In another preclinical study, tumors of mice with patient xenografts showed a depletion of desmoplastic stroma when treated with nab-paclitaxel [32]. The reduction in stromal content was accompanied by dilated blood vessels in the tumor milieu. The effect of stromal depletion was highest when mice were treated with the combination of nab-paclitaxel and gemcitabine. The authors concluded that the reduction in tumor stroma and the increase in vascularization facilitated the delivery of gemcitabine to the tumors. In fact, intratumor concentrations of gemcitabine increased by 2.8-fold with a combination treatment of nab-paclitaxel and gemcitabine compared to mice treated with gemcitabine alone [32].

A neoadjuvant trial supports the concept of stromal depletion by nab-paclitaxel [53]. 16 patients with operable pancreatic cancer received 2 cycles of nab-paclitaxel and gemcitabine before surgical resection. $50 \%$ of patients achieved a $>75 \%$ decrease in circulating CA 19-9 tumor marker and a response demonstrated by fluorodeoxyglucose (FDG)-PET. 7 of 12 patients who completed treatment and were operated had major pathological regressions. There was also a significant decrement in tumor stiffness as measured by endoscopic ultrasound elastography. This went in parallel with a clear change in the architecture of the tumor stroma as indicated by markedly disorganized collagen and a very low density of cancer-associated fibroblasts, which was not observed in the untreated or conventionally treated control groups. A preclinical co-clinical study in a mouse model showed that these effects were specific of nab-paclitaxel and not gemcitabine [53].

\section{Synergism of Nab-Paclitaxel and Gemcitabine through Paclitaxel-Induced Decrease in Gemcitabine- Metabolizing Enzyme Cytidine Deaminase}

In animal models, the combination of gemcitabine and nabpaclitaxel increased gemcitabine concentration in plasma and tumor tissues [52]. The increased intratumoral gemcitabine levels were a result of a marked decrease in cytidine deaminase, the primary gemcitabine-metabolizing enzyme. This was attributed to nab-paclitaxel as experiments had demonstrated that paclitaxel actually reduced cytidine deaminase protein in cultured cells [54].

\section{Discussion}

For over a decade, the standard of care offered patients with metastatic PDAC had a proven, yet modest clinical benefit. Patients had no options available that would have offered a clinically relevant longer OS than the $~ 5-6$ months with gemcitabine monotherapy. 2 new combinations have now demonstrated superior efficacy over gemcitabine. FOLFIRINOX and the combination of nab-paclitaxel and gemcitabine significantly reduced the risk of death and provided a clinically relevant prolongation of OS [20,49]. This is an important leap in the management of advanced pancreatic cancer. Currently, patients can be offered 4 potential first-line treatment options. However, there are differences to consider as illustrated by the details of the pivotal trials outlined in table 2, bearing in mind that cross-trial comparisons do have limitations. Patients in the trial reported by Burris et al. [8] had a rather poor performance status. Only $72 \%$ in the trial reported by Burris et al. [8] and 77\% in the trial reported by Moore et al. [19] actually had metastatic disease. Patients in the 2 phase III trials published recently represent a selected patient population with a rather good performance status and low serum bilirubin levels $[20,49]$. There are no data for patients older than 76 years from the FOLFIRINOX trial [20], while $10 \%$ of patients in the trial published by von Hoff were older than 75 years [49]. The table shows that clinically relevant advances have been achieved with FOLFIRINOX and nabpaclitaxel/gemcitabine. The armamentarium of therapeutic combination chemotherapy in first-line treatment of metastatic disease has thus expanded substantially. Actually, the challenge lies in developing an individualized treatment for each patient that includes the best integration and sequencing of agents or combinations. For decision making, the clinician may consider patient-related factors such as age, Eastern Cooperative Oncology Group (ECOG) performance status, co-morbidity, and the patient's attitude towards toxicity, as well as the tumor characteristics and other clinical parameters such as serum bilirubin and the extent of disease (potential operability?).

There are implications beyond the optimal treatment choice for first-line metastatic PDAC. A real step ahead can 
Table 2. Overview of selected patient characteristics, efficacy and safety parameters from landmark phase III trials in chemotherapy-naive advanced pancreatic cancer patients

$\begin{array}{llll}\begin{array}{l}\text { Gemcitabine } \\ \text { vs. 5-fluorouracil }\end{array} & \begin{array}{l}\text { Gemcitabine + erlotinib } \\ \text { vs. gemcitabine }\end{array} & \begin{array}{l}\text { FOLFIRINOX } \\ \text { vs. gemcitabine }\end{array} & \begin{array}{l}\text { Nab-paclitaxel } \\ + \text { gemcitabine }\end{array} \\ & \mathrm{N}=569[19] & \mathrm{N}=342[20] & \begin{array}{l}\text { vs. gemcitabine } \\ \mathrm{N}=861[49-51,55]\end{array} \\ \end{array}$

\begin{tabular}{|c|c|c|c|c|c|}
\hline \multicolumn{6}{|l|}{ Patient characteristics } \\
\hline \multicolumn{2}{|c|}{ Median age, years (range) } & $62(37-79)$ & $64(38-84)$ & $61(25-76)$ & $62(27-86)$ \\
\hline \multirow[t]{3}{*}{ Stage } & II & $14 \%$ & $0 \%$ & $0 \%$ & $0 \%$ \\
\hline & III & $14 \%$ & $24 \%$ & $0 \%$ & $0 \%$ \\
\hline & IV & $72 \%$ & $77 \%$ & $100 \%$ & $100 \%$ \\
\hline \multirow[t]{2}{*}{ Performance status } & $\mathrm{KPI} \leq 70 / \mathrm{ECOG} 2$ & $70 \%($ KPI $50-70)$ & $19 \%$ & $1 \%$ & $8 \%$ (KPI 60-70) \\
\hline & KPI $80-100 /$ ECOG $0-1$ & $30 \%($ KPI 80-90) & $81 \%$ & $99 \%$ & $93 \%$ \\
\hline \multirow{2}{*}{\multicolumn{2}{|c|}{ Bilirubin eligibility threshold }} & $\leq 2.0 \mathrm{mg} / \mathrm{dl}$ & adequate hepatic & $\leq 1.5$ times upper & $\leq$ upper limit \\
\hline & & & function & limit of normal range & of normal range \\
\hline \multicolumn{6}{|l|}{ Efficacy } \\
\hline ORR, \% & & 5.4 (evaluable pts) & 8.6 (evaluable pts) & 31.6 (ITT) & 23 (ITT) \\
\hline \multirow[t]{5}{*}{ Best response, \% } & $\mathrm{CR}$ & 0 & n.a. & 0.6 & $<1$ \\
\hline & PR & 5.4 & n.a. & 31.0 & 23 \\
\hline & $\mathrm{SD}$ & 39 & 48.9 & 38.6 & 27 \\
\hline & $\mathrm{PD}[\%]$ & n.r. $\left(55.4^{\mathrm{a}}\right)$ & n.r. $\left(42.5^{\mathrm{a}}\right)$ & 15.2 & 20 \\
\hline & Not evaluable & n.a. & n.a. & 14.6 & 30 \\
\hline \multicolumn{2}{|c|}{ Median PFS, months (PFS comparator) } & $2.08^{\mathrm{b}}(0.92)$ & $3.75(3.55)$ & $6.4(3.3)$ & $5.5(3.7)$ \\
\hline \multicolumn{2}{|c|}{ HR for PFS (vs. gemcitabine) } & n.a. & 0.77 & 0.47 & 0.69 \\
\hline \multicolumn{2}{|c|}{ Median OS, months (OS comparator) } & $5.65(4.41)$ & $6.24(5.91)$ & $11.1(6.8)$ & $8.7(6.6)$ \\
\hline \multicolumn{2}{|c|}{ HR for OS (vs. gemcitabine) } & n.a. & 0.82 & 0.57 & 0.72 \\
\hline \multicolumn{6}{|c|}{ Selected grade $3 / 4$ toxicities } \\
\hline \multicolumn{2}{|c|}{ Neutropenia } & 25.9 & 24 & 45.7 & 38 \\
\hline \multicolumn{2}{|l|}{ Febrile neutropenia } & n.r. & n.r. & 5.4 & 3 \\
\hline \multicolumn{2}{|l|}{ Fatigue } & n.r. & n.r. & 23.6 & 17 \\
\hline \multicolumn{2}{|l|}{ Vomiting } & 12.7 & 15 & 14.5 & 6 \\
\hline \multicolumn{2}{|l|}{ Diarrhea } & 1.6 & 6 & 12.7 & 6 \\
\hline \multicolumn{2}{|l|}{ Sensory neuropathy } & 0 & n.r. & $9.0^{\mathrm{c}}$ & $17^{\mathrm{d}}$ \\
\hline \multicolumn{6}{|c|}{ 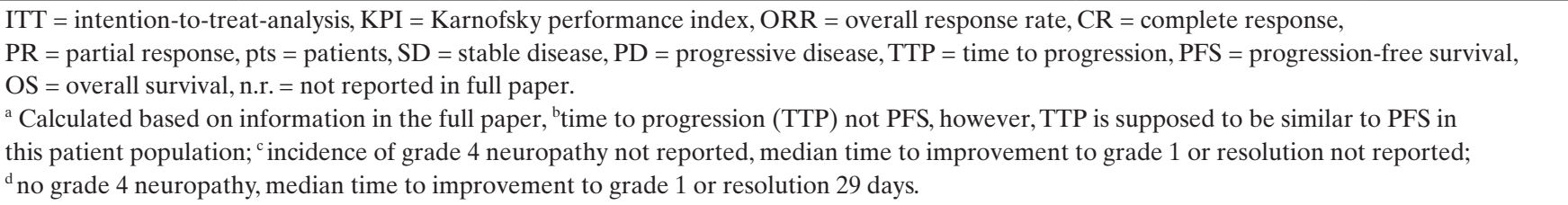 } \\
\hline
\end{tabular}

only be achieved when more patients are cured. Therefore, the value of the combination of nab-paclitaxel and gemcitabine as a neoadjuvant and adjuvant treatment of pancreatic cancer should be explored. A trial evaluating nab-paclitaxel and gemcitabine in the adjuvant setting is underway. Due to the demonstrated increased PFS and OS, the new options provide a realistic basis to consider treatment sequences or second-line options for patients with metastatic PDAC.

Data regarding the proposed mechanisms of action are not yet conclusive. However, the preclinical and clinical data of nab-paclitaxel support the general therapeutic concept of stromal depletion in pancreatic cancer as one to evaluate fur- ther. The data also suggest that SPARC should be explored as a predictive biomarker.

\section{Disclosure Statement}

The authors received medical writing support in the preparation of this manuscript from Dr. Susanne Hell, funded by Celgene Corporation. The authors were fully responsible for all content and editorial decisions for this manuscript. S.A., M.G. and H.O. participated in advisory boards for Celgene Corporation. T.S. received lecture fees from the Celgene Corporation.

\section{References}

1 Jemal A, Bray F, Center MM, et al.: Global cancer statistics. CA Cancer J Clin 2011, 61:69-90.

2 Krebs in Deutschland 2007/2008, Eine gemeinsame Veröffentlichung des Robert Koch-Instituts und der Gesellschaft der epidemiologischen Krebsregister in Deutschland e. V. 8. Berlin, Robert-Koch-Institut, 2012.
Hassan MM, Bondy ML, Wolff RA, et al.: Risk factors for pancreatic cancer: Case control study. Am J Gastroenterol 2007;102:2696-2707.

4 Shi C, Hruban RH, Klein AP: Familial pancreatic cancer. Arch Pathol Lab Med 2009;133:365-374.

5 Tersmette AC, Petersen GM, Offerhaus GJ, et al.: Increased risk of incident pancreatic cancer among first-degree relatives of patients with familial pancreatic cancer. Clin Cancer Res 2001;7:738-744.
6 Neesse A, Frese KK, Chan DS, et al.: SPARC independent drug delivery and antitumour effects of nab-paclitaxel in genetically engineered mice. Gut. 2013 doi: 10,1136/gutjnl-2013-305559. [Epub ahead of print].

7 Seufferlein T, Bachet JB, Van Cutsem E, et al.: ESMO-ESDO Clinical Practice Guidelines for diagnosis, treatment and follow-up. Ann Oncol 2012; 23 Suppl 7:vii33-40. 
8 Burris HA 3rd, Moore MJ, Andersen J, et al.: Improvements in survival and clinical benefit with gemcitabine as first-line therapy for patients with advanced pancreas cancer: A randomized trial. J Clin Oncol. 1997;15:2403-2413.

-9 Cunningham D, Chau I, Stocken DD, et al.: Phase III randomized comparison of gemcitabine versus gemcitabine plus capecitabine in patients with advanced pancreatic cancer. J Clin Oncol 2009;27: 5513-5518.

10 Heinemann V, Quietzsch D, Gieseler F, et al.: Randomized phase III trial of gemcitabine plus cisplatin compared with gemcitabine alone in advanced pancreatic cancer. J Clin Oncol 2006;24: 3946-3952.

11 Louvet C, Labianca R, Hammel P, et al.: Gemcitabine in combination with oxaliplatin compared with gemcitabine alone in locally advanced or metastatic pancreatic cancer: Results of a GERCOR and GISCAD phase III trial. J Clin Oncol 2005;23: 3509-3516.

$\checkmark 12$ Reni M, Cordio S, Milandri C, et al.: Gemcitabine versus cisplatin, epirubicin, fluorouracil, and gemcitabine in advanced pancreatic cancer: A randomised controlled multicentre phase III trial. Lancet Oncol 2005;6:369-376.

$\checkmark 13$ Ko AH, Youssoufian H, Gurtler J, et al.: A phase II randomized study of cetuximab and bevacizumab alone or in combination with gemcitabine as firstline therapy for metastatic pancreatic adenocarcinoma. Invest New Drugs 2012;30:1597-1606.

14 Kindler HL, Niedzwiecki D, Hollis D, et al.: Gemcitabine plus bevacizumab compared with gemcitabine plus placebo in patients with advanced pancreatic cancer: phase III trial of the Cancer and Leukemia Group B (CALGB 80303). J Clin Oncol. 2010;28:3617-3622.

15 Oettle H, Richards DA, Ramanathan RK, et al.: A phase III trial of pemetrexed plus gemcitabine versus gemcitabine in patients with unresectable or metastatic pancreatic cancer. Ann Oncol 2005; 16:1639-1645.

16 Berlin JD, Catalano P, Thomas JP, et al.: Phase III study of gemcitabine in combination with fluorouracil versus gemcitabine alone in patients with advanced pancreatic carcinoma: Eastern Cooperative Oncology Group trial E2297. J Clin Oncol 2002;20:3270-3275.

17 Rocha Lima CM, Green MR, Rotche R, et al.: Irinotecan plus gemcitabine results in no survival advantage compared with gemcitabine monotherapy in patients with locally advanced or metastatic pancreatic cancer despite increased tumor response rate. J Clin Oncol 2004;22:3776-3783.

18 Abou Alfa GK, Letourneau R, Harker G, et al.: Randomized phase III trial of exatecan and gemcitabine compared with gemcitabine alone in untreated advanced pancreatic cancer. J Clin Oncol 2006;24:4441-4447.

19 Moore MJ, Goldstein D, Hamm J, et al.: Erlotinib plus gemcitabine compared with gemcitabine alone in patients with advanced pancreatic cancer: A phase III trial of the National Cancer Institute of Canada Clinical Trials Group. J Clin Oncol. 2007;25:1960-1966.

20 Conroy T, Desseigne F, Ychou M, et al.: FOLFIRINOX versus gemcitabine for metastatic pancreatic cancer. N Engl J Med. 2011;364:18171825.

21 Heinemann V, Reni M, Ychou, et al.: Tumourstroma interactions in pancreatic ductal adenocarcinoma: Rationale and current evidence for new therapeutic strategies. Cancer Treat Rev 2014;40: 41 Ibrahim NK, Desai N, Legha S, et al.: Phase I and $118-128$.

22 Li X, Ma Q, Xu Q, et al.: Targeting the Cancerstroma interaction: A potential aproach for pancreatic cancer treatment. Curr Pharm Des 2012;18: 2404-2415.

23 Olive KP, Jacobetz MA, Davidson CJ, et al.: Inhibition of Hedgehog signaling enhances delivery of chemotherapy in a mouse model of pancreatic cancer. Science 2009;324:1457-1461.

24 Garber K: Stromal depletion goes on trial in pancreatic cancer. J Natl Cancer Institute 2010;102:448-450.

25 Pandol S, Edderkaoui M, Gukovsky I, et al.: Desmoplasia of pancreatic ductal adenocarcinoma. Clin Gastroenterol Hepatol 2009;7:44-47.

26 Motamed K: SPARC (osteonectin/BM-40). Int J Biochem Cell Biol 1999;31:1363-1366.

27 Podhajcer OL, Benedetti LG, Girotti MR, et al.: The role of the matricellular protein SPARC in the dynamic interaction between the tumor and the host. Cancer Metastasis Rev 2008;27:691-705.

28 Arnold SA, Brekken RA: SPARC: A matricellular regulator of tumorigenesis. J Cell Commun Signal 2009;3:255-273.

29 Guweidhi A, Kleeff J, Adwan H, et al.: Osteonectin influences growth and invasion of pancreatic cancer cells. Ann Surg 2005;242:224-234.

30 Desai N, Piacente M, Liu X, et al.: Effect of plasma SPARC on outcome in cancer models, in: Proceedings of the 46th Annual Meeting of the American Society of Clinical Oncology 2010; abstract 10600.

31 Watkins G, Douglas-Jones A, Bryce R, et al.: Increased levels of SPARC (osteonectin) in human breast cancer tissues and its association with clinical outcomes. Prostaglandins Leukot Essent Fatty Acids 2005;72:267-262.

32 Von Hoff DD, Ramanathan RK, Borad MJ, et al.: Gemcitabine plus nab-paclitaxel is an active regimen in patients with advanced pancreatic cancer: a phase I/II trial. J Clin Oncol. 2011;29:4548-4554.

33 Infante JR, Matsubayashi H, Sato N, et al.: Peritumoral fibroblast SPARC expression and patient outcome with resectable pancreatic adenocarcinoma. J Clin Oncol. 2007;25:319-325.

34 Neuzillet C, Tijeras-Raballand A, Cros J, et al.: Stromal expression of SPARC in pancreatic adenocarcinoma. Cancer Metastasis Rev. 2013;32:585602.

35 Kiessling F, Fink C, Hansen M, et al.: Magnetic resonance imaging of nude mice with heterotransplanted high-grade squamous cell carcinomas. Invest Radiol 2002;37:193-198.

-36 Chlenski A, Guerrero LJ, Salwen HR, et al.: Secreted protein acidic and rich in cysteine is a matrix scavenger chaperone. PLoS One 2011;6: e23880.

37 Abraxane SmPC, version January 14th 2013.

38 Desai N: Nab technology: A drug delivery platform utilising endothelial gp60 receptor-based transport and tumour-derived SPARC for targeting. Drug Delivery Report Winter 2007/2008:37-41.

39 Desai N, Trieu V, Yao Z, et al.: Increased antitumor activity, intratumor paclitaxel concentrations, and endothelial cell transport of cremophor-free, albumin-bound paclitaxel, ABI- 007, compared with cremophor-based paclitaxel. Clin Cancer Res 2006; 12:1317-1324.

40 Gardner ER, Dahut WL, Scripture CD, et al.: Randomized crossover pharmacokinetic study of solvent-based paclitaxel and nab-paclitaxel. Clin Cancer Res. 2008;14:4200-4205. pharmacokinetic study of ABI-007, a Cremophorfree, protein-stabilized, nanoparticle formulation of paclitaxel. Clin Cancer Res 2002;8:1038-1044.

42 Gradishar W, Tjulandin S, Davidson N, et al.: Phase III trial of nanoparticle albumin-bound paclitaxel compared with polyethylated castor oil-based paclitaxel in women with breast cancer. J Clin Oncol 2005;23:7794-7803.

43 Socinski MA, Bondarenko I, Karaseva NA, et al.: Weekly nab-paclitaxel in combination with carboplatin versus solvent-based paclitaxel plus carboplatin as first-line therapy in patients with advanced non-small-cell lung cancer: Final results of a phase III trial. J Clin Oncol 2012;30:2055-2062.

44 Hersh E, Del Vecchio M, Brown M, et al.: Phase 3 , randomized, open-label, multicenter trial of nabpaclitaxel (nab-P) versus dacarbazine (DTIC) in previously untreated patients with metastatic malignant melanoma (MMM). Pigment Cell Melanoma Res 2012;25:863.

45 Hosein PJ, de Lima Lopes G Jr, Pastorini VH, et al.: A phase II trial of nab-paclitaxel as secondline therapy in patients with advanced pancreatic cancer. Am J Clin Oncol 2013;36:151-156.

46 Zhang DS, Wang DS, Wang ZQ, et al.: Phase I/II study of albumin-bound nab-paclitaxel plus gemcitabine administered to Chinese patients with advanced pancreatic cancer. Cancer Chemother Pharmacol 2013;71:1065-1072.

47 Tsimberidou AM, Ye Y, Wheler J, et al.: A phase I study of hepatic arterial infusion of nab-paclitaxel in combination with intravenous gemcitabine and bevacizumab for patients with advanced cancers and predominant liver metastases. Cancer Chemother Pharmacol 2013;71:955-963.

48 Ko AH, Truong TG, Kantoff E, et al.: A phase I trial of nab-paclitaxel, gemcitabine, and capecitabine for metastatic pancreatic cancer. Cancer Chemother Pharmacol 2012;70:875-881.

49 Von Hoff DD, Ervin T, Arena F, et al.: Increased survival in pancreatic cancer with nab-paclitaxel plus gemcitabine. N Engl J Med 2013;369:16911703.

50 Von Hoff DD, Ervin TJ, Arena FP, et al.: Results of a randomized phase III trial (MPACT) of weekly $n a b$-paclitaxel plus gemcitabine versus gemcitabine alone for patients with metastatic adenocarcinoma of the pancreas with PET and CA19-9 correlates. J Clin Oncol 2013;31(suppl; abstr 4005).

51 Abraxane ${ }^{\circledR}$ Prescription information, version of October 2013.

52 Awasthi N, Zhang C, Schwarz AM, et al.: Comparative benefits of Nab-paclitaxel over gemcitabine or polysorbate-based docetaxel in experimental pancreatic cancer. Carcinogenesis 2013;34:23612369.

53 Alvarez R, Musteanu M, Garcia-Garcia E, et al.: Stromal disrupting effects of nab-paclitaxel in pancreatic cancer. Br J Cancer 2013;109:926-933.

54 Frese KK, Neesse A, Cook N, et al.: Nab-paclitaxel potentiates gemcitabine activity by reducing cytidine deaminase levels in a mouse model of pancreatic cancer. Cancer Discov 2012;2:260-269.

55 Goldstein D, El Maraghi RH, Hammel P et al.: Updated survival from a randomized phase III trial (MPACT) of nab-paclitaxel plus gemcitabine versus gemcitabine alone for patients (pts) with metastatic adenocarcinoma of the pancreas. J Clin Oncol 2014;32(suppl 3; abstr 178). 